\title{
Circulating Fibroblast Growth Factor-23 Increases Following Intermittent Parathyroid Hormone (1-34) in Postmenopausal Osteoporosis: Association with Biomarker of Bone Formation
}

\author{
Geeta Hampson
}

Published online: 31 March 2011

(C) Springer Science+Business Media, LLC 2011

We thank Mahon and Casey for their interest in our paper [1]. We agree with them that our study population is not representative of the usual population treated with bisphosphonates. As described in our article, the patients either were intolerant to or had had an unsatisfactory response to oral bisphosphonates and therefore qualified for treatment with PTH analogue (teriparatide) as set out by the NICE guidance. This would explain the lack of suppression seen at baseline in the bone turnover markers.

We would like to clarify that we did not set out to investigate the role of FGF-23 as a marker of osteoporosis. We wanted to address, firstly, the unresolved issues surrounding the role of PTH on FGF-23 production/secretion and, secondly, whether FGF-23 is involved in bone formation, independently of its established role in phosphate homeostasis. In a previous study, PTH infusion acutely increased circulating FGF-23 in healthy men [2]. As reported in our study, we found an increase in FGF-23 concentrations during treatment with teriparatide in postmenopausal women with osteoporosis. We also found a significant correlation between the skeletal anabolic effect of intermittent PTH, as assessed by P1NP, and increases in FGF-23. These findings point to the possibility of a new mechanistic pathway for PTH's anabolic actions on bone. We acknowledge that these are preliminary findings and that the small sample number and study design preclude any definitive conclusions. We agree that further studies of the role of FGF-23 as a marker in osteoporosis should be undertaken in larger populations.

\section{References}

1. Sridharan M, Cheung J, Moore AE, Frost ML, Fraser WD, Fogelman I, Hampson G (2010) Circulating fibroblast growth factor-23 increases following intermittent parathyroid hormone (1-34) in postmenopausal osteoporosis: association with biomarker of bone formation. Calcif Tissue Int 87(5):398-405

2. Burnett-Bowie SA, Henao MP, Dere ME, Alaee H, Leder BZ (2009) Effects of h PTH (1-34) infusion on circulating serum phosphate, 1, 25-dihydroxyvitamin D and FGF-23 levels in healthy men. J Bone Miner Res 24:1681-1685
G. Hampson $(\bowtie)$

Department of Chemical Pathology/Metabolic Bone Clinic,

Guy's and St Thomas' NHS Foundation Trust,

London SE1 7EH, UK

e-mail: geeta.hampson@kcl.ac.uk 